\title{
Size-dependent inelastic behavior of particle-reinforced metal-matrix composites
}

\author{
L.H. Dai *, Z. Ling, Y.L. Bai \\ State Key Laboratory of Nonlinear Mechanics (LNM), Institute of Mechanics, Chinese Academy of Sciences, Beijing 100080, PR China
}

Received 21 January 2000; received in revised form 9 August 2000; accepted 14 November 2000

\begin{abstract}
The strengthening behavior of particle-reinforced metal-matrix composites (MMCp) is primarily attributed to the dislocation strengthening effect and the load-transfer effect. To account for these two effects in a unified way, a new hybrid approach is developed in this paper by incorporating the geometrically necessary dislocation strengthening effect into the incremental micromechanical scheme. By making use of this hybrid approach, the particle-size-dependent inelastic deformation behavior of MMCp is given. Some comparisons with the available experimental results demonstrate that the present approach is satisfactory. (C) 2001 Elsevier Science Ltd. All rights reserved.
\end{abstract}

Keywords: A. Metal-matrix composites (MMCs); B. Nonlinear behavior; C. Deformation; Size effect

\section{Introduction}

Particle-reinforced metal-matrix composites (MMCp) have the potential to provide desirable mechanical properties including high specific stiffness, specific strength, and high creep resistance. This suite of properties makes MMCp strong candidates for use in aerospace, defense and automobile applications. As a class of microstructuresensitive materials, the mechanical behavior of MMCp depends significantly on the reinforcement pattern. Therefore, the understanding of the relationship between the strengthening behavior and the microstructure is a critical issue in developing advanced MMCp.

It is generally recognized that two types of strengthening may occur in MMCp: direct and indirect. Direct strengthening results from load transfer from the metal matrix to the reinforcing particle whereas indirect strengthening results from the influence of reinforcement on matrix microstructure or deformation mode. One example of indirect strengthening is the dislocation strengthening induced by the deformation mismatch between the reinforcement and the matrix. To explain the load-transfer effect, various continuum models including

\footnotetext{
* Corresponding author. Tel.: +86-10-62545533, ext. 2117; fax: +86-10-62561284.

E-mail address: 1hdai@Inm.imech.ac.cn (L.H. Dai).
}

the shear-lag theory [1-3] and the homogenization methods [4-9] have been proposed during the past several decades. The shear-lag theory assumes that the load transfer occurs between a high-aspect-ratio reinforcement and the matrix by means of shear stresses at the particle-matrix interface. By this mechanism the particle can act as a "reinforcement" to bear some of the load. The original model [1] only accounts for the shear transfer of load along the interphase parallel to the applied load. Modifications have subsequently been made by Nardone and Prewo [2] and Piggot [3] for the case of a small aspect ratio of reinforcements. By contrast with the shear-lag theory, the homogenization method addresses mainly on the stress allocation between the reinforcement and the matrix in a volume average sense, so that the load-transfer effect can be included. Once the stress concentration factors of the constituents are known, the overall strengthening behavior of MMCp can be determined by the analytical approach for linear composites and by the finite-element method [4,5], the secant modulus method [6,7] or incremental method [8,9] for non-linear composites.

It is noted that continuum models lead to a dependence of deformation behavior on the volume fraction of the reinforcing particle but not on particle size. However, several recent experimental studies [10-12] have clearly displayed a significant effect of the particle size on 
inelastic behavior of MMCp. To understand the particle-size effect, dislocation models $[13,14]$ and the strain gradient plasticity theory [15] have been developed in the past. Nevertheless, these models lead to a smaller increase in the yield strength than the observations in experiments due to the fact that only indirect strengthening was involved. Recently, some strain gradient-dependent bounds on the overall behavior of linear and nonlinear composites have been developed by Smyshlyaev and Fleck $[16,17]$ for addressing the size effect in composites.

In view of the aforementioned observations, a new hybrid micromechanical approach, namely a marriage of the geometrically-necessary dislocation model and the incremental micromechanical scheme, is developed in this paper. In this hybrid approach, both direct and indirect strengthening effects are included. A comparison with the available experimental results demonstrate that the present new approach is quite satisfactory.

\section{Dislocation strengthening behavior}

It is well known that the microstructure and properties of the composite matrix or in situ matrix may be significantly different from those of the unreinforced matrix alloy. The presence of particles induces an inhomgeneous deformation pattern in MMCp even if the composite is subjected to uniform loading (as shown in Fig. 1) and high dislocation density in the composite matrix, thus leading to a higher yield strength in the composite matrix. The increase in the dislocation density in the composite matrix is assumed to be mainly due to the elastic modulus (EM) mismatch and the coefficient of thermal expansion (CTE) mismatch between the matrix and the reinforcement. The dislocations generated by EM mismatch and CTE mismatch can be considered as two examples of geometrically necessary dislocations of Ashby [18].

\subsection{EM mismatch}

Consider a MMCp subjected to a compressive loading, as is shown in Fig. 2a. Subscripts "m", "p", and "c" stand for the matrix, the particle and the composite respectively. To determine the geometrically necessary dislocation density, an idea similar to Eshelby's equivalent inclusion principle [19] is adopted. Firstly, imagine that all particles in the MMCp sample of Fig. 2a are replaced by the matrix material, thus particles turning into "matrix spheres". So, the whole body of this matrix sample will experience a uniform deformation $\varepsilon$ when the sample is subjected to a uniform compressive loading. In this case, those "matrix spheres" will be distorted into "matrix ellipsoids". However, in the real MMCp sample such a distortion will not be allowed to take place because of the existence of the reinforcing particles.

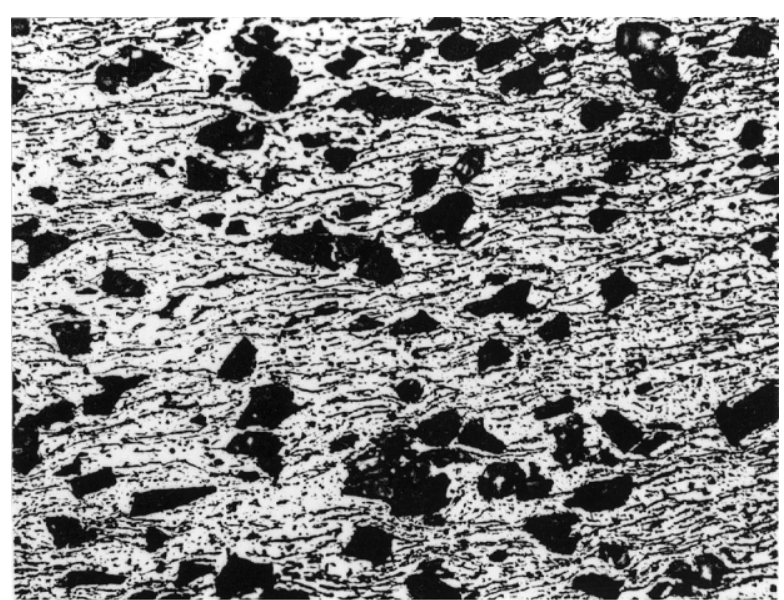

a) $d_{p}=13 \mu \mathrm{m}$

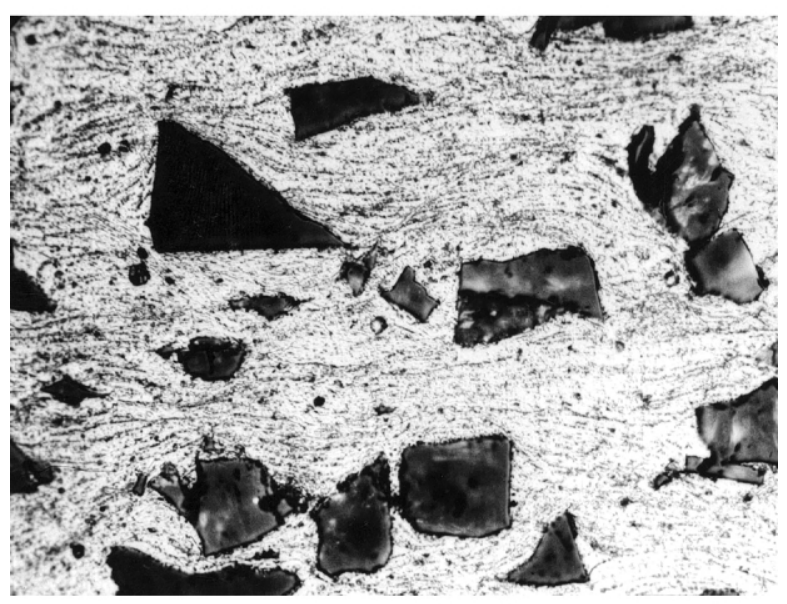

b) $d_{p}=37 \mu \mathrm{m}$

Fig. 1. Inhomogeneous plastic deformation pattern in $\mathrm{SiCp} / 2124 \mathrm{Al}$ composites under compressive loading [10]: (a) $13 \mu \mathrm{m}$; (b) $37 \mu \mathrm{m}$.

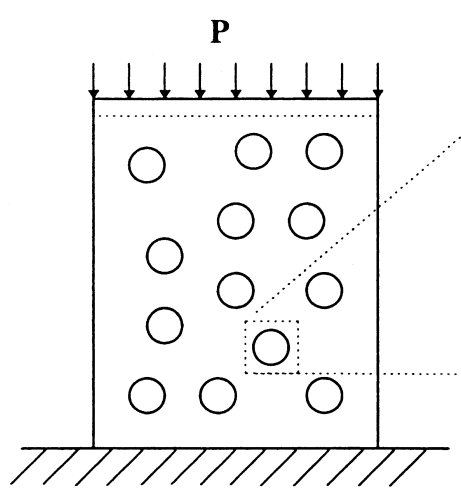

(a)

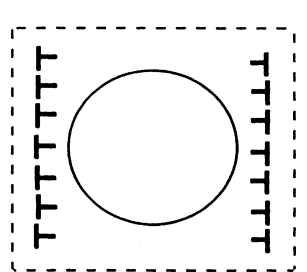

(b)
Fig. 2. Configuration and EM-mismatch dislocation model for MMCp under compressive loading: (a) configuration; (b) dislocation model.

Hence, a lot of geometrically necessary dislocations must be stored near the surfaces of particles for accommodating this distortion deformation (Fig. 2b). According to the deformation-geometry condition, the number of the geometrically necessary dislocation loops imposed on 
the surfaces of particles to accommodate the mismatch of plastic deformation $n^{\mathrm{EM}}$ is approximately estimated by

$n^{\mathrm{EM}} b=\varepsilon d_{\mathrm{p}}$

where $b$ is Burgers vector, $d_{\mathrm{p}}$ is the diameter of particles. Assume the volume fraction of particles is denoted by $f_{\mathrm{p}}$, then the total number of particles $N_{\mathrm{p}}$ is

$N_{\mathrm{p}}=\frac{6 f_{\mathrm{p}}}{\pi d_{\mathrm{p}}^{3}}$

If the length for each dislocation loop is taken as $\pi d_{p}$, then the geometrically necessary dislocation density for EM mismatch is

$\rho_{\mathrm{G}}^{\mathrm{EM}}=\frac{6 f_{\mathrm{p}}}{b d_{\mathrm{p}}} \varepsilon$

\subsection{CTE mismatch}

When a MMCp is quenched from $T_{0}$ to $T_{\mathrm{q}}$, resulting in a temperature change $\Delta T=T_{0}-T_{\mathrm{q}}$, mismatch strain $\varepsilon_{\mathrm{CTE}}$ will be induced in particles and it is given by

$\varepsilon_{\mathrm{CTE}}=\left(C_{\mathrm{m}}-C_{\mathrm{p}}\right) \Delta T=\Delta C \cdot \Delta T$

where $C_{\mathrm{m}}, C_{\mathrm{p}}$ are CTE of the matrix and the particle, respectively. For a real hard-particle MMC, such a deformation mismatch will be constrained by the particle. Thus, in order to accommodate this thermal mismatch deformation, amounts of geometrically necessary loops will be imposed around the surfaces of particles. The number of the dislocation loops imposed on each particle for CTE mismatch $n^{\mathrm{CTE}}$ can be approximately estimated by

$n^{\mathrm{CTE}} b A_{\mathrm{p}}=\Delta V_{\mathrm{p}}$

where $A_{\mathrm{p}}=\frac{1}{4} \pi d_{\mathrm{p}}^{2}$ is the area of dislocation loop, $\Delta V_{\mathrm{p}}=$ $\frac{1}{2} \pi d_{\mathrm{p}}^{3} \cdot \Delta C \cdot \Delta T$ is the volume change due to CTE mismatch. Thus, $n^{\mathrm{CTE}}$ can be written as

$n^{\mathrm{CTE}}=\frac{2 d_{\mathrm{p}} \Delta C \cdot \Delta T}{b}$

The length for each loop is assumed to be $\pi d_{\mathrm{p}}$. So, the total geometrically dislocation density due to CTE mismatch is given by

$\rho_{\mathrm{G}}^{\mathrm{CTE}}=\frac{12 f_{\mathrm{p}} \Delta C \cdot \Delta T}{b d_{\mathrm{p}}}$

It is seen from Eqs. (3) and (7) that the geometrically necessary dislocation density to accommodate EM mismatch and CTE mismatch for the small particle is higher than that for the large particle.

The increased dislocation density in MMCp leads to the in situ matrix being of higher yield strength over the unreinforced metal matrix. For this case, the yield strength of the in situ matrix is given by

$\sigma_{\mathrm{my}}=\sigma_{\mathrm{m} 0}+\Delta \sigma$

where $\sigma_{\mathrm{my}}, \sigma_{\mathrm{m} 0}$ are the yield strength of the in situ matrix and the unreinforced matrix, respectively. While $\Delta \sigma$ the total increment in yield stress of the in situ matrix and can be estimated by [20]

$\Delta \sigma=\sqrt{\left(\Delta \sigma_{\mathrm{EM}}\right)^{2}+\left(\Delta \sigma_{\mathrm{CTE}}\right)^{2}}$

Here, $\Delta \sigma_{\mathrm{EM}}, \Delta \sigma_{\mathrm{CTE}}$ are determined by Taylor dislocation strengthening relation:

$\Delta \sigma_{\mathrm{EM}}=\sqrt{3} \alpha \mu_{\mathrm{m}} b \sqrt{\rho_{\mathrm{G}}^{\mathrm{EM}}}$

$\Delta \sigma_{\mathrm{CTE}}=\sqrt{3} \beta \mu_{\mathrm{m}} b \sqrt{\rho_{\mathrm{G}}^{\mathrm{CTE}}}$

where $\mu_{\mathrm{m}}$ is the shear modulus of the matrix, two dislocation strengthening coefficients $\alpha$ and $\beta$ are taken respectively to be 0.5 and 1.25 in subsequent calculations.

\section{Incremental micromechanical scheme}

Instead of adopting the shear lag theory, the incremental micromechanical scheme is used here to characterize the load transfer effect. The incremental selfconsistent scheme was first developed by Hill [21] and Hutchinson [22] to predict the overall mechanical behavior of polycrystalline aggregates. In extending this method to the inclusion based composites, some researchers, e.g. Ponte Castaneda and Sequet [23], have found that the method gives predictions that violate rigorous bounds. However, in our recent extension, this phenomenon was not found and our extended incremental method gives slightly softer predictions than those of the secant methods [9]. The full information on our incremental method and its applications to MMCp with progressive interface damage will be discussed in another paper. Here, only the principal idea of the incremental scheme is outlined.

Consider a MMCp consisting of elastic spherical particles perfectly bonded to a nonlinear metal matrix. The local constitutive behavior of constituents can be characterized by a strain potential $U(\sigma)$ :

$\varepsilon=\frac{\partial U(\sigma)}{\partial \sigma}$ 
This constitutive model is commonly used to represent a number of nonlinear mechanical phenomena, including time-independent plastic deformation (i.e. deformation theory of plasticity) and time-dependent viscous deformation (e.g. high temperature creep) of metals. In the first case, $\sigma$ and $\varepsilon$ are the infinitesimal stress and strain tensors. In the second case, $\sigma$ and $\varepsilon$ should be identified with the Cauchy stress and Eulerian strain rate, respectively. For simplicity, attention is focused on a class of isotropic materials for which $U(\sigma)$ reads as

$U(\sigma)=\frac{1}{2 k} \sigma_{\mathrm{m}}^{2}+\varphi\left(\sigma_{\mathrm{e}}\right)$

where $k$ is bulk modulus, $\sigma_{\mathrm{m}}=\operatorname{tr}(\sigma) / 3$ and $\sigma_{\mathrm{e}}=$ $(3 s: s / 2)^{1 / 2}$ are the first two invariants of $\sigma$, while $s$ is the deviatoric stress. By differentiation with respect to time, the local constitutive relation (12) can be expressed in the incremental form:

$\dot{\varepsilon}=M_{\mathrm{t}}(\sigma): \dot{\sigma}$

where $M_{\mathrm{t}}(\sigma)$ is the local tangent compliance tensor for the constituents. Following the spectral decomposition used by Ponte Castaneda [24], $M_{\mathrm{t}}(\sigma)$ can be written as

$M_{\mathrm{t}}(\sigma)=\frac{1}{3 k} J+\frac{1}{2 \mu_{t}} K+\frac{1}{\lambda} F$

where

$J=\stackrel{(2)}{I} \otimes \stackrel{(2)}{I} / 3 \quad K=\stackrel{(4)}{I}-\stackrel{(2)}{I} \otimes \stackrel{(2)}{I} / 3$

$F=\hat{s} \otimes \hat{s} \quad \hat{s} \equiv s / \sigma_{e}$

while

$k=\sigma_{\mathrm{m}} /[3 \partial U(\sigma) / \partial \operatorname{tr}(\sigma)] \quad \mu_{t}=\sigma_{\mathrm{e}} / 3^{\prime}\left(\sigma_{\mathrm{e}}\right)$

$\lambda=(4 / 9)\left[\sigma_{\mathrm{e}} /\left(\sigma_{\mathrm{e}} \varphi^{\prime \prime}\left(\sigma_{\mathrm{e}}\right)-\varphi^{\prime}\left(\sigma_{\mathrm{e}}\right)\right]\right.$

$\varphi^{\prime}\left(\sigma_{\mathrm{e}}\right)=\partial \varphi\left(\sigma_{\mathrm{e}}\right) / \partial \sigma_{\mathrm{e}} \quad \varphi^{\prime \prime}\left(\sigma_{\mathrm{e}}\right)=\partial^{2} \varphi\left(\sigma_{\mathrm{e}}\right) / \partial^{2} \sigma_{\mathrm{e}}$

(2) $\stackrel{(4)}{I}$ and

where $I$ and $I$ are the second-rank and the fourth-rank unit tensors, respectively.

In general, the local tangent compliance tensor $M_{\mathrm{t}}(\sigma)$ is anisotropic and varies from one point to another, a simplified assumption is made and constant tangent tensor is considered in nonlinear matrix phase. However, $M_{\mathrm{t}}(\sigma)$ is the stress-state dependent and evaluated at some effective stress for the matrix phase. It is noted that the tangent compliance tensor defined by (15) depend not only on the first two stress invariants (equivalently, $\sigma_{\mathrm{m}}$ and $\sigma_{\mathrm{e}}$ ) but also on $F=\hat{s} \otimes \hat{s}$ which cannot be completely determined by the first two stress invariants. Hence, the currently-defined tangent compliance tensor can, at least partly, reflect the effect of the third stress invariant. It is this point that differs from the widely-used secant method [25] where the effect of the third stress can not be incorporated. For some cases, such as power-law materials reinforced by rigid particles, Lee and Mear [26] and Suquet and Ponte Castaneda [27] have demonstrated that the effect of the third stress invariant cannot be ignored.

The overall stress-strain behavior of MMCp can be characterized by the following incremental constitutive relation:

$\dot{\bar{\varepsilon}}=\bar{M}_{\mathrm{t}}(\bar{\sigma}): \dot{\bar{\sigma}}$

where $\bar{\sigma}, \bar{\varepsilon}$ are the macroscopic stress and strain for the composite. While $\bar{M}_{\mathrm{t}}$ is the overall or effective tangent compliance tensor of the composite. Usually, determining $\bar{M}_{\mathrm{t}}$ is very difficult due to involving in solving the anisotropic-matrix inclusion problem. In our present scheme, $\bar{M}_{\mathrm{t}}$ is determined by the generalized self-consistent Mori-Tanaka method which was recently developed by Dai et al. [28]

$$
\begin{aligned}
& \bar{M}_{\mathrm{t}}=M_{\mathrm{t}}^{(\mathrm{m})}+f_{\mathrm{p}}\left(M^{(\mathrm{p})}-M_{\mathrm{t}}^{(\mathrm{m})}\right): \\
& {\left[\left(1-f_{\mathrm{p}}\right) M_{\mathrm{t}}^{(\mathrm{m})^{-1}}: H: M^{(\mathrm{p})}+f_{\mathrm{p}} I\right]^{(4)}}
\end{aligned}
$$

where $M_{\mathrm{t}}^{(\mathrm{m})}, M^{(\mathrm{p})}$ are the tangent compliance tensors of the matrix and the particle, respectively. While the inclusion/matrix interaction strain concentration tensor $H$ is defined by

$H=\stackrel{(4)}{I}+P:\left(M^{(\mathrm{p})^{-1}}-M_{\mathrm{t}}^{(\mathrm{m})^{-1}}\right)$

where $P$ is related to Eshelby tensor of particle $S$ by $P=S: M_{\mathrm{t}}^{(\mathrm{m})}, S$ was given by Mura [29] for the anisotropic-matrix inclusion problem.

The overall behavior of nonlinear MMCp can be modeled by a series of incremental steps. In each incremental step, the nonlinear composite will be characterized by a linear one so that the average stress in nonlinear matrix phase can be determined by making use of the linear homogeneous approach. In this paper we consider the case that the matrix phase is nonlinear and the spherical particle remains elastic in the course of whole deformation procedure. Since the tangent compliance of nonlinear material depends on the current stress state in the considered material, the average stress in the matrix at each incremental step should be determined. Once the average stress of the matrix is determined, the local tangent compliance tensor of the matrix phases can be obtained from Eq. (15). Then, the overall tangent compliance tensor of the composite can 
be estimated by (19) for each incremental step. Hence, the overall stress-strain curve for the composite can be obtained point by point using the incremental constitutive relation (18).

\section{Size-dependent inelastic behavior}

To predict the particle size-dependent behavior of MMCp, a new hybrid approach, namely incorporating the dislocation strengthening effect into the incremental micromechanical scheme, is presented in this section. Although the dislocation model and strain gradient theory can predict particle size and volume fractiondependent behavior of MMCp, our recent study [15], however, has demonstrated that these models lead to a smaller increase in yield and flow strength than was observed in experiment of Ling et al. [10,11], which is shown in Fig. 3. On the other hand, the incremental micromechanical scheme does not directly predict a particle size dependence on deformation behavior, although a dependence on volume fraction is predicted. However, recent experimental results have clearly demonstrated that both particle size and volume fraction exert an influence [10-12]. Therefore, we hope the present hybrid approach can give a reasonable prediction of the particle size-dependent behavior of MMCp.

The key point involved in this hybrid approach lies in replacing the yield stress of the unreinforced metal matrix $\left(\sigma_{\mathrm{m} 0}\right)$ by that of the in situ matrix $\left(\sigma_{\mathrm{my}}\right)$, as shown in Eq. (8). To check the utility of the present hybrid approach, comparisons with the available experimental results are made here.

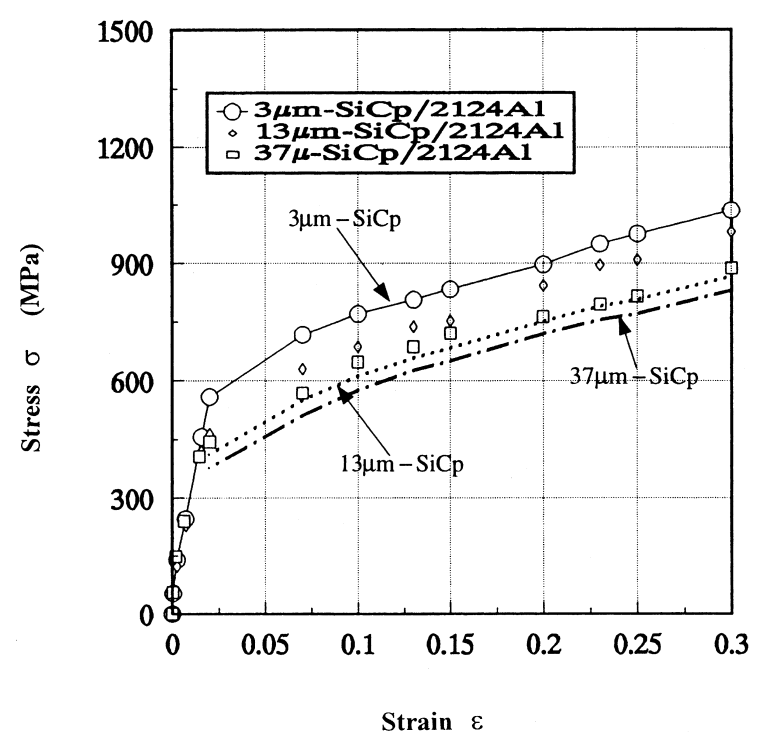

Fig. 3. A comparison of uniaxial compressive stress-strain curves predicted by the strain gradient law [15] with the experimental data [10] for $\mathrm{SiCp} / 2124 \mathrm{Al}$ composite.
To investigate the particle size effect, a series of uniaxial compression tests of $2124 \mathrm{Al}$ and $2124 \mathrm{Al}$ reinforced with $17 \%$ volume fraction of 3,13 , and $37 \mu \mathrm{m} \mathrm{SiC} \mathrm{particles}$ were carried out by Ling et al. [10,11]. Recently, Lloyd [12] performed uniaxial tension tests on A356 aluminum alloy and $15 \%$ Vol. SiCp/A356 composites with two particle sizes, 7.5 and $16 \mu \mathrm{m}$. Both these two types of experiments displayed a pronounced increase in yield and flow strength of the composites with decreased particle size. The pertinent mechanical and physical properties for both kinds of materials are listed in Table 1. While the uniaxial stress-strain behavior for both $2124 \mathrm{Al}$ and A356 alloy can be modeled by an empirical power law such as the Ramburg-Osgood relation

$\varepsilon=\frac{\sigma}{E_{\mathrm{m}}}+\xi \frac{\sigma_{\mathrm{m} 0}}{E_{\mathrm{m}}}\left(\frac{\sigma}{\sigma_{\mathrm{m} 0}}\right)^{1 / n}$

equivalently, this uniaxial stress-strain relation can be characterized by the following nonlinear strain potential $U(\sigma)$ :

$U(\sigma)=\frac{1}{2 k_{\mathrm{m}}} \sigma_{\mathrm{m}}^{2}+\frac{1}{6 \mu_{\mathrm{m}}} \sigma_{\mathrm{e}}^{2}+\xi \frac{n}{n+1} \frac{\sigma_{\mathrm{m} 0}^{2}}{E_{\mathrm{m}}}\left(\frac{\sigma_{\mathrm{e}}}{\sigma_{\mathrm{m} 0}}\right)^{\frac{n+1}{n}}$

where Ramberg-Osgood factor $\xi$ is taken as $3 / 7$, $k_{\mathrm{m}}, \mu_{\mathrm{m}}, E_{\mathrm{m}}$ are the bulk, shear, and Young's moduli of the matrix, respectively. $n$ is the strain hardening exponent, $\sigma_{\mathrm{m} 0}$ is the yield stress of the unreinforced matrix and should be replaced by that of the in situ matrix $\sigma_{\text {my }}$ in the present hybrid approach. Table 2 shows the parameters in Ramberg-Osgood equation, $n, \sigma_{\mathrm{m} 0}$, which are obtained by fitting Eq. (21) to the unreinforced matrix alloy flow curves given in Refs. [10] and [12].

Figs. 4 and 5 present the comparison of the predicted uniaxial stress-strain curves by the hybrid approach with the experimental results of Ling et al. $[10,11]$ and Lloyd [12], respectively. From Figs. 3-5, we can see that

Table 1

Material properties

\begin{tabular}{llccc}
\hline Parameter & Unit & $2124 \mathrm{Al}$ & $\mathrm{A} 356$ & $\mathrm{SiC}$ \\
\hline Young's modulus & $\mathrm{GPa}$ & 72 & 70 & 427 \\
Poisson ratio & & 0.31 & 0.33 & 0.17 \\
CTE & $\times 10^{-6} /{ }^{\circ} \mathrm{C}$ & 24 & 23.6 & 4.3 \\
Burgers vector & $\mathrm{nm}$ & 0.283 & 0.283 & \\
\hline
\end{tabular}

Table 2

Ramberg-Osgood parameters

\begin{tabular}{llc}
\hline Matrix alloy & $n$ & $\sigma_{\mathrm{m} 0}(\mathrm{MPa})$ \\
\hline $2124 \mathrm{Al}$ & 0.089 & 354 \\
A356-T6 & 0.110 & 208 \\
A356-T4 & 0.213 & 85 \\
\hline
\end{tabular}




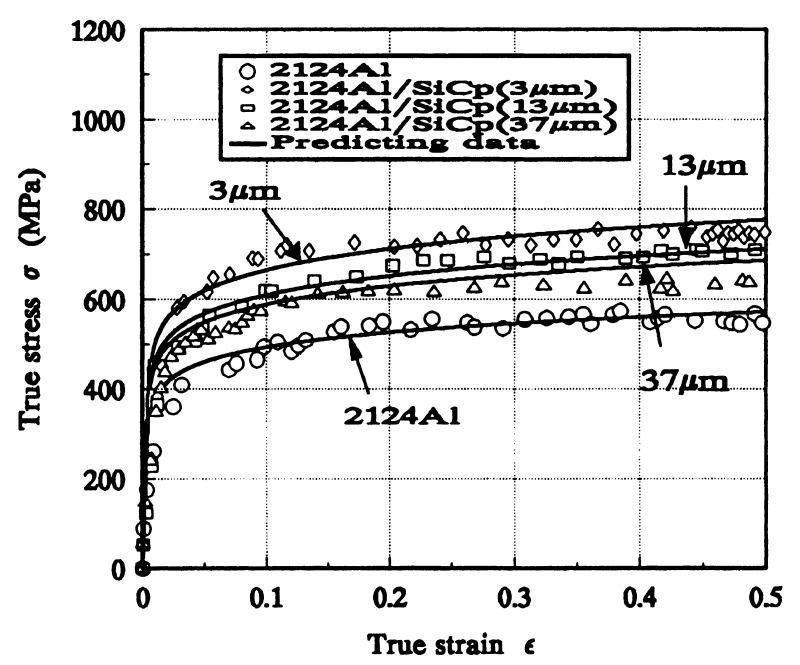

Fig. 4. Uniaxial compression stress-strain behavior for $\mathrm{SiCp} / 2124 \mathrm{Al}$ composites (symbol points: experimental data [10]; solid lines: predicting data).
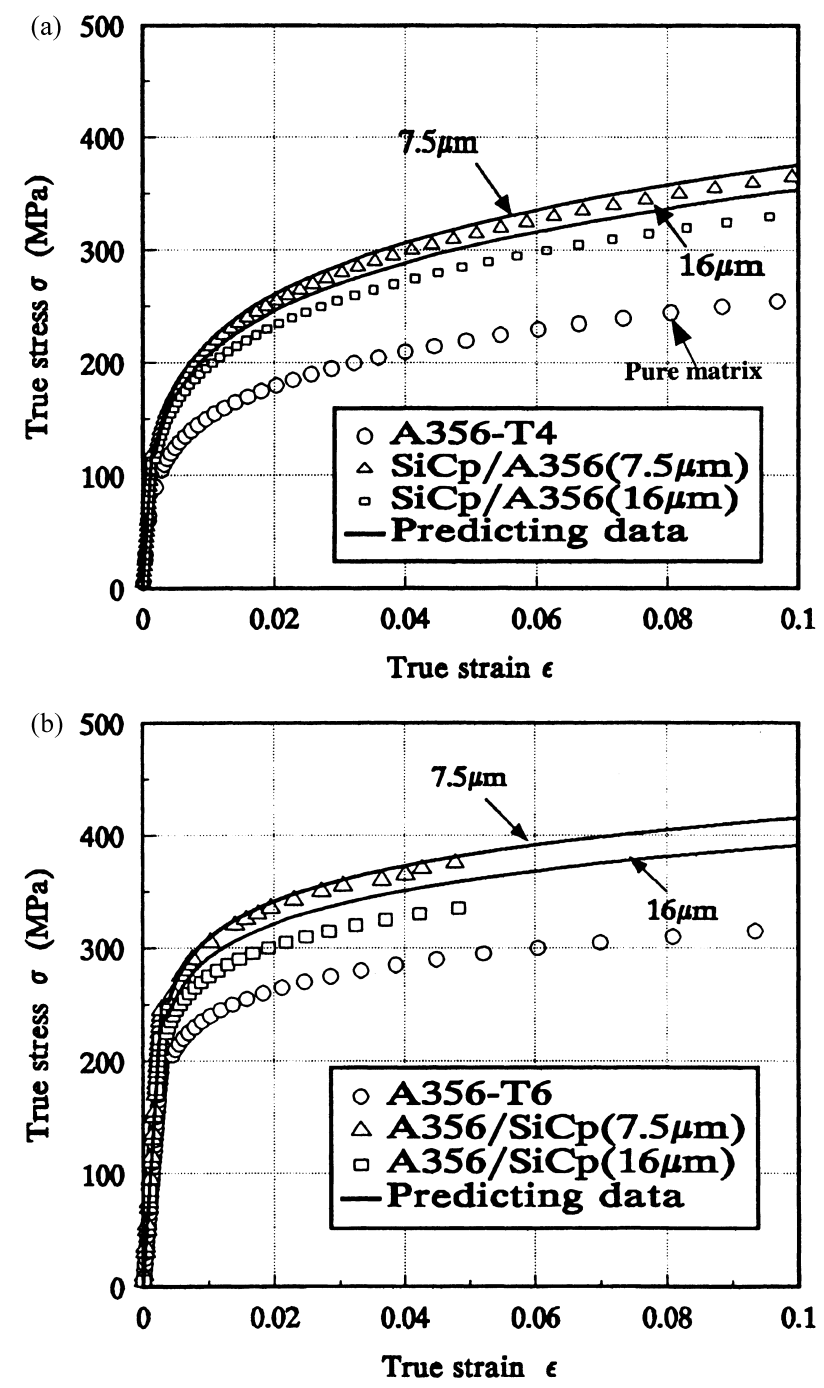

Fig. 5. Uniaxial tension stress-strain behavior for $\mathrm{SiCp} / \mathrm{A} 356$ composites (symbol points: experimental data [12]; solid lines: predicting data): (a) $\mathrm{SiCp} / \mathrm{A} 356-\mathrm{T} 4 \mathrm{Al}$ composite; (b) $\mathrm{SiCp} / \mathrm{A} 356-\mathrm{T} 6 \mathrm{Al}$ composite. the present hybrid approach gives a much better prediction (Figs. 4 and 5) than that of the strain gradient theory (Fig. 3). The reason for this point is that the strain gradient theory only considers the indirect strengthening (dislocation strengthening) whereas the present hybrid approach includes both direct strengthening (load transfer from the matrix to the reinforcing particles) and indirect strengthening. This tells us that both direct and indirect strengthening effects should be considered in order to predict accurately the size-dependent inelastic behavior of MMCp. For large particle case, $d_{\mathrm{p}}>10 \mu \mathrm{m}$, a slight deviation of the theoretical results from the experimental data can be found. This may be due to the intrinsic limitation of the dislocation strengthening model, because the dislocation strengthen behavior has been turned out to be modeled effectively for small particle by many researchers. Undoubtedly, the further attempts are still necessary to understand such a deviation phenomenon. In spite of these, the comparison demonstrates that the present hybrid approach is satisfactory on the whole.

\section{Conclusions}

By incorporating the dislocation strengthening effect into the incremental micromechanical scheme, a new hybrid approach is developed in this paper for characterizing the particle size-dependent inelastic deformation behavior of MMCp. In the present hybrid approach, both direct strengthening (load transfer) and indirect strengthening (dislocation strengthening) effects are incorporated. Comparisons with the available experimental results demonstrate that the present hybrid approach is satisfactory.

\section{Acknowledgements}

The authors gratefully acknowledge the financial support of this research by the National Natural Science Foundation of China (project numbers: 19902017 and 19891180) and Major project of Chinese Academy of Sciences (KJ-951-1-201)

\section{References}

[1] Cox HL. The elasticity and strength of the paper and other fibrous materials. Br J Appl Phys 1952;3:73-9.

[2] Nardone VC, Prewo KM. On the strength of discontimuous silicon carbide reinforced aluminum composites. Scripta Metall 1986;20:43-8.

[3] Piggot MR. Load-bearing fibre composite. Oxford: Pergamon Press, 1990.

[4] Christman T, Needleman A, Suresh S. An experimental and numerical study of deformation in MMCs. Acta Metall 1989;37:3029-50. 
[5] Bao G, Hutchinson JW, McMeeking RM. Particle reinforcement of ductile matrices against plastic flow and creep. Acta Metall 1991;39:1871-82.

[6] Qiu YP, Weng GJ. A theory of plasticity for porous materials and particle-reinforced composites. J Appl Mech 1992;59:261-8.

[7] Hu G. A method of plasticity for general aligned spheroidal void and fiber-reinforced composites. Int J Plasticity 1996;12:439-49.

[8] Tohgo K, Chou TW. Incremental theory of particle reinforced composite including debonding damage. JSME Int J 1996;39:389401.

[9] Dai LH. Effective elastoplastic theory of multiphase composites. Beijing: Peking University Postdoctoral Research Work Report, 1998.

[10] Ling Z, Luo L, Dodd B. Experimental study on the formation of shear bands and effect of microstructure in $2124 \mathrm{Al} / \mathrm{SiCp}$ composite under dynamic compression. J de Physique 1994;C3:453.

[11] Ling Z. Deformation behavior and microstructure effect in 2124Al/SiCp composite. J Comp Mater 2000;34:101-15.

[12] Lloyd DJ. Particle reinforced aluminum and magnesium matrix composites. Int Mater Rev 1994;39:1-23.

[13] Kamat SV, Rollett AD, Hirth JP. Plastic deformation in Al alloy matrix-alumina particulate composites. Scripta Metall 1991;25:2732.

[14] Miller WS, Humphreys FJ. Strengthening mechanisms in particulate metal matrix composites. Scripta Metall 1991;25:33-8.

[15] Dai LH, Ling Z, Bai YL. A strain gradient strengthening law for particle reinforced metal matrix composites. Scripta Mater 1999;41:245-51.

[16] Smyshlyaev VP, Fleck NA. Bounds and estimates for linear composites with strain gradient effects. J Mech Phys Solids 1994;42:1851-82.
[17] Smyshlyaev VP, Fleck NA. Bounds and estimates for the overall plastic behaviour of composites with strain gradient effects. Proc R Soc Lond A 1995;451:795-810.

[18] Ashby MF. The deformation plastically nonhomgeneous alloys. Phil Mag 1970;21:399.

[19] Eshelby JD. The determination of the field of an ellipsoidal inclusion and related problems. Proc Roy Soc 1957;A241:37696.

[20] Clyne TW, Withers PJ. Introduction to metal matrix composites. Cambridge: Cambridge University Press, 1993.

[21] Hill R. Continuum micromechanics of elastoplatic polycrystals. J Mech Phys Solids 1965;13:89-101.

[22] Hutchinson JW. Elatic-plastic behavior of polycrystalline metals. Proc Roy Soc London 1970;A319:247-72.

[23] Ponte Castaneda P, Suquet P. Nonlinear composites. Advances in Appl Mech 1998;34:172-302.

[24] Ponte Castaneda P. Exact second-order estimates for the effective mechanical properties of nonlinear composite materials. J Mech Phys Solids 1996;44:827-62.

[25] Tandon GP, Weng GJ. A theory of particle reinforced plasticity. ASME J Appl Mech 1988;55:126-35.

[26] Lee B, Mear WE. Effect of inclusion shape on the stiffness of nonlinear two-phase composites. J Mech Phys Solids 1991;39:627-49.

[27] Suquet P, Ponte Castaneda P. Small-contrast perturbation expansions for the effective properties of nonlinear composites. CR Acad Sci Paris Ser 1993;II317:1515-22.

[28] Dai LH, Huang ZP, Wang R. A generalized self-consistent MoriTanaka scheme for prediction of the effective moduli of hybrid multiphase particulate composites. Polymer Composites 1998;19:506-13.

[29] Mura T. Micromechanics of defects in solids. Nijhoff, 1987. 